\title{
Polycyclic Aromatic Hydrocarbons Residues in Sandstorm Depositions in Beijing, China
}

\author{
S. Fu $\cdot$ K. Li $\cdot$ X. J. Xia $\cdot$ X. B. Xu
}

Received: 21 April 2008/Accepted: 22 August 2008/Published online: 5 September 2008

(C) Springer Science+Business Media, LLC 2008

\begin{abstract}
This study was conducted to determine the concentration of 16 polycyclic aromatic hydrocarbons (PAHs) in sandstorm depositions in Beijing, China. The PAH concentrations in 13 samples collected in Beijing ranged from 0.18 to $3.52 \mu \mathrm{g} \mathrm{g}^{-1}$. Analysis of the sources of contamination revealed that the PAHs were derived from a coal combustion source, although various effects of traffic emissions were also observed. Furthermore, the PAH levels in Beijing tended to be higher in the southeast. Finally, the nemerow composite index revealed that the degree of pollution in the sandstorm depositions varied widely among sampling sites.
\end{abstract}

Keywords China - Deposition P Polycyclic aromatic hydrocarbons $\cdot$ Sandstorm

Polycyclic aromatic hydrocarbons (PAHs) are an important class of organic pollutants that have received considerable attention due to their carcinogenic and mutagenic properties (Blackburn and Kellard 1986). As a result, sixteen un-substituted PAHs are included in the US Environmental Protection Agency (EPA) list of priority pollutants that require monitoring. For these reasons, many studies have recently been conducted to evaluate PAHs in the

S. Fu · K. Li · X. J. Xia · X. B. Xu (

State Key Laboratory of Environmental Chemistry

and Ecotoxicology, Research Center for Eco-Environmental

Sciences, Chinese Academy of Sciences, 18 Shuangqing Road,

Haidian District, P.O. Box 2871, Beijing 100085,

People's Republic of China

e-mail: xuxb1006@sina.com

S. Fu

e-mail: fu_shan@sina.com environment (Durjava et al. 2007). PAHs are produced by the incomplete combustion of organic materials or during pyrolisis (Bernal-Martinez et al. 2007). Therefore, PAHs are discharged into the atmosphere as both gases and aerosols, after which they are transported through the atmosphere until they are ultimately deposited in the terrestrial environment. Atmospheric deposition is likely a predominant source of PAH loading into the terrestrial environment (Franz et al. 1991). Atmospheric depositions are important factors in controlling the regional and global distribution of PAHs, as well as assessing their impact on ecosystems. Furthermore, sandstorms that distribute a great number of particles are unique atmospheric occurrences that occur frequently in northern China. For example, northern China experienced eleven sandstorms in 2006. One of these sandstorms covered approximately $13 \%$ of China and deposited about 330,000 tons of sand/dust in Beijing (Han et al. 2007). Northeast China is one of the areas most affected by sandstorms. Beijing, which is located in northeast China, is one of the most densely populated cities in the world, with more than 15 million residents. Sandstorms have seriously contaminated Beijing's atmosphere and have had a direct influence on public health. However, the sources of human exposure to PAHs remain poorly understood and few studies have been conducted to evaluate the contamination caused by sandstorm particles. Furthermore, the level of pollutants in deposition from sandstorms has not been well characterized. Because PAHs can easily accumulate in particles, contamination by sandstorm deposition can have a direct impact on public health by increasing the risk of human exposure via inhalation, ingestion or direct skin contact (Blackburn and Kellard 1986). Therefore, further information regarding the presence of PAHs in sandstorm deposition is needed to better understand their environmental behavior and source-sink relationships. 
This study was conducted to evaluate the concentration of 16 PAHs in sandstorm depositions in Beijing. Because there is little data regarding PAHs in environmental media in China, we conducted this study to determine both the concentration and profile of PAHs in sandstorm deposition samples obtained from urban areas of Beijing. The primary objective of this study was to identify the possible sources of pollution in the sandstorm depositions of Beijing.

\section{Materials and Methods}

A serious sandstorm that developed in a Mongolian cyclone blew over Beijing on April 16, 2006 (Han et al. 2007). During the sampling period, the climate in Beijing was dominated by temperate semi-wet monsoon conditions, and the mean temperature was $14^{\circ} \mathrm{C}$. We obtained sandstorm deposition samples from 13 urban sites in Beijing, China (Fig. 1) on April 17, 2006. The area of the sampling region was $750 \mathrm{~km}^{2}$. Samples were collected from platforms $\left(0.5-1.0 \mathrm{~m}\right.$ high with an area of and $\left.3 \mathrm{~m}^{2}\right)$ during the daytime (between 8:00 A.M. and 6:00 P.M. $\left.\left[10 \mathrm{~h} \mathrm{day}^{-1}\right]\right)$ when there was no precipitation. Composite samples comprised of three subsamples obtained at each sampling site were then transferred into precleaned amber glass bottles and stored at $4^{\circ} \mathrm{C}$ until they were extracted, which was usually done within $72 \mathrm{~h}$. We then analyzed the particle size distributions of the sandstorm deposition samples using a Laser Particle Size Analyzer (Mastersizer 2000; Malvern Co., United Kingdom).

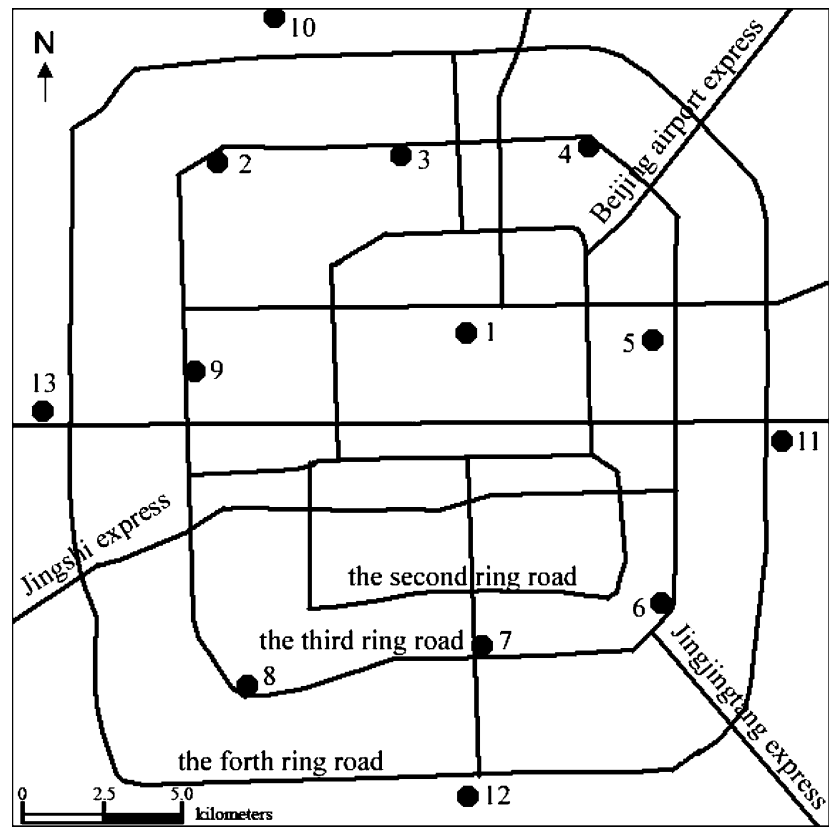

Fig. 1 Sampling sites of sandstorm deposition in urban areas of Beijing
A mixed PAH standard solution $\left(1,000 \mu \mathrm{g} \mathrm{mL}^{-1}\right)$ that included naphthalene (Na), acenaphthylene (Acy), acenaphthene (Ace), fluorene ( $\mathrm{Fl}$ ), phenanthrene (Phe), anthracene (An), fluoranthene (Flu), pyrene (Pyr), benz[a]anthracene $(\mathrm{BaA})$, chrysene (Chr), benzo[b]fluoranthene $(\mathrm{BbF})$, benzo[k]fluoranthene $(\mathrm{BkF})$, benzo[a]pyrene $(\mathrm{BaP})$, indeno [1,2,3-cd]pyrene (InP), dibenz[a,h]anthracene (DBA), and benzo[g,h,i]perylene (BghiP) was purchased from Sigma Aldrich, St. Louis, MO (Milwaukee, WI, USA). In addition, 2-flurobiphenyl (2-FBP), which was used as a surrogate standard, was purchased from Supelco (Bellefonte, PA, USA). The standards were diluted to the desired concentration using isooctane and then used as working standards. Silica gel (100-200 mesh) was purchased from Qingdao Haiyang Chemical Co. (Qindao, China) and then activated in a drying oven at $550^{\circ} \mathrm{C}$ for $6 \mathrm{~h}$. Anhydrous sodium sulfate (Beijing Chemical Factory, Beijing, China) was heated at $600^{\circ} \mathrm{C}$ for $12 \mathrm{~h}$ prior to being used to eliminate organic contamination. All solvents used were of pesticide grade and purchased from J. T. Baker (Phillipsburg, NJ, USA).

Five grams of each sample were weighed accurately and then ground into a free-flowing powder with anhydrous sodium sulfate with a mortar and pestle. The samples were then extracted with $30 \mathrm{~mL}$ of hexane/acetone $(1: 1, \mathrm{v} / \mathrm{v})$ by ultrasound at 3,000g for $4 \mathrm{~min}$, after which the samples were centrifuged and the supernatant removed. This process was repeated three times, after which the extracts were purified using a chromatograph column $(30 \mathrm{~cm} \times 10 \mathrm{~mm}$ i.d.) containing $6 \mathrm{~g}$ of activated silica gel and $2 \mathrm{~g}$ of anhydrous sodium sulfate. The fraction containing the PAHs was then eluted using $25 \mathrm{~mL}$ hexane/dichloromethane $(3: 2, v / v)$, after which the solvent was evaporated to $100 \mu \mathrm{L}$ under a gentle $\mathrm{N}_{2}$ stream and stored for subsequent analysis.

The PAHs were analyzed using an Agilent 6890 gas chromatograph equipped with a flame ionization detector. Separation was performed on a $30 \mathrm{~m}$ DB-5MS (30 $\mathrm{m} \times 0.25 \mathrm{~mm}$ i.d., $0.25-\mu \mathrm{m}$ film thickness) capillary column. The temperature of the injector and detector were $280^{\circ} \mathrm{C}$ and $300^{\circ} \mathrm{C}$, respectively. After the sample was injected, the GC column was maintained at $70^{\circ} \mathrm{C}$ for $2 \mathrm{~min}$, then ramped at $4^{\circ} \mathrm{C} \mathrm{min}{ }^{-1}$ to $280^{\circ} \mathrm{C}$ and held at this temperature for $10 \mathrm{~min}$. The total run time was $54.5 \mathrm{~min}$.

To confirm the PAH results, selected typical samples were evaluated using an Agilent 6890 series gas chromatograph coupled to an Agilent 5973 mass spectrometer using an electron impact ionization source in the selected ion monitoring mode. In EI mode, the MS source temperature was $230^{\circ} \mathrm{C}$, the transfer line was $300^{\circ} \mathrm{C}$ and the electron energy was $70 \mathrm{eV}$. Gas chromatographic separation was then conducted under the conditions described above.

In addition, a laboratory method control group was analyzed to demonstrate that the samples were not affected 
by interference or cross-contamination. Furthermore, a procedural blank was run in parallel for every set of 6 samples to check for interference and cross-contamination. Moreover, duplicate samples were analyzed in the laboratory as an additional quality control tool to ensure the validity of the results. The instrument stability and relative response factor variance were also measured by analyzing the calibration standard solutions during the analysis each sample batch.

Identification of PAHs was confirmed, after which the concentrations were measured using an external quantification standard consisting of known amounts of the target compound. The following quality control criteria were used to ensure correct identification of the target compound: (a) The GC retention times matched those of the standard compounds within $\pm 0.05 \mathrm{~min}$. (b) The signal-to-noise ratio was greater than 3:1. The limits of detection for the 16 PAHs were defined as a signal-to-noise ratio greater than three times the average baseline variation and were within the range of $1.80-2.53 \mathrm{ng} \mathrm{g}^{-1}$ (dry weight). The matrix spike recoveries of the 16 PAHs were within the range of $76-106 \%$, with a standard deviation of $10 \%$. The recovery of the 2-FBP in all samples was in the range of 70-90\%.

\section{Results and Discussion}

PAHs were detected in all sandstorm deposition samples. The total concentration of PAHs (the sum of all 16 PAHs) ranged from 0.18 to $3.52 \mu \mathrm{g} \mathrm{g}^{-1}$ (median: $0.40 \mu \mathrm{g} \mathrm{g}^{-1}$, dry weight) (Table 1). Few studies have been conducted on sandstorm deposition to date; however, it is known that sandstorm deposition directly influences surface soil.
Therefore, we compared the concentrations of PAHs in the samples collected for this study to soil concentrations that have been previously reported. In China, the PAH concentrations in soil vary widely among cities and regions, and occasionally within the same city (e.g., Beijing) (Caia et al. 2008). Specifically, the sum concentrations of PAHs ( $\Sigma$ PAHs) in soils collected from different regions of China range from not detectable (Hong Kong) to $27.8 \mathrm{mg} \mathrm{kg}^{-1}$ (Beijing), with a mean value of $1.2( \pm 1.1) \mathrm{mg} \mathrm{kg}^{-1}$ (mean \pm S.D.) (Caia et al. 2008). These values were much lower than the PAH concentrations in urban soils from other countries, including New Orleans, USA (3.731 $\mu \mathrm{g} \mathrm{g}^{-1}$ as median) (Mielke et al. 2001), Tokushima, Japan (0.611 $\mathrm{g} \mathrm{g} \mathrm{g}^{-1}$ as mean) (Yang et al. 2002) and Estonia (2.200 $\pm 1.396 \mu \mathrm{g} \mathrm{g}^{-1}$ ) (Trapido 1999).

The composition of PAHs produced by different sources varies significantly. Therefore, the characteristic spectrum of PAHs can be used to identify their sources. The ratios of the sum of the major combustion specific compounds ( $\Sigma \mathrm{COMB}$, including Flu, Pyr, BaA, Chr, BbF, BkF, BaP, InP and Bghip) to the sum of the 16 EPA-PAHS ( $\Sigma$ COMB/ $\Sigma$ EPA-PAHs), as well the $\mathrm{An} /(\mathrm{An}+\mathrm{Phe})$ and $\mathrm{Flu} /(\mathrm{Flu}+\mathrm{Pyr})$ ratios are generally used to identify the sources of PAHs (Table 1) (Zhang et al. 2004). In general, combustion induces the production of relatively higher concentrations of $\Sigma \mathrm{COMB}$; therefore a large proportion of $\Sigma C O M B$ is characteristic of PAHs that originated from combustion processes (Zhang et al. 2004). Conversely, an An/(An + Phe) ratio of $<0.10$ generally indicates that the PAHs were formed by petroleum combustion while an $\mathrm{An} /(\mathrm{An}+\mathrm{Phe})$ ratio $>0.10$ indicates that the source of the PAHs was coal combustion (Budzinski et al. 1997). Furthermore, cruel oil samples generally produce a Flu/(Flu + Pyr) ratio of $<0.40$ while coal combustion

Table 1 Total PAHs, low molecular weight PAHs (2-3-ring PAHs, LMW PAHs), high molecular weight PAHs (4-6-ring PAHs, HMW PAHs), selected PAH ratios and the nemerow composite index $(p)$ values of sandstorm deposition samples

\begin{tabular}{|c|c|c|c|c|c|c|c|c|}
\hline $\begin{array}{l}\text { Sampling } \\
\text { sites }\end{array}$ & $\begin{array}{l}\text { Total PAHs } \\
\left(\mu \mathrm{g} \mathrm{g}^{-1}\right)\end{array}$ & $\begin{array}{l}\text { LMW PAHs } \\
\left(\mu \mathrm{g} \mathrm{g}^{-1}\right)\end{array}$ & $\begin{array}{l}\text { HMW PAHs } \\
\left(\mu \mathrm{g} \mathrm{g}^{-1}\right)\end{array}$ & $\begin{array}{l}\Sigma \mathrm{COMB} / \Sigma \mathrm{EPA}- \\
\mathrm{PAHs}\end{array}$ & $\begin{array}{l}\mathrm{An} / \\
(\mathrm{An}+\mathrm{Phe})\end{array}$ & $\begin{array}{l}\text { Flu/ } \\
(\text { Flu + Pyr })\end{array}$ & $\begin{array}{l}\mathrm{BaP} / \\
\text { BghiP }\end{array}$ & $p$ \\
\hline 1 & 0.29 & 0.03 & 0.26 & 0.79 & 0.04 & 0.33 & 0.69 & 1.39 \\
\hline 2 & 0.54 & 0.10 & 0.44 & 0.75 & 0.25 & 0.34 & 0.81 & 1.40 \\
\hline 3 & 0.18 & 0.60 & 0.12 & 0.64 & 0.07 & 0.52 & 1.02 & 0.79 \\
\hline 4 & 0.31 & 0.12 & 0.20 & 0.59 & 0.28 & 0.52 & 0.48 & 0.39 \\
\hline 5 & 3.52 & 0.87 & 2.66 & 0.68 & 0.15 & 0.44 & 1.21 & 10.46 \\
\hline 6 & 0.19 & 0.03 & 0.16 & 0.79 & 0.82 & 0.46 & 5.58 & 2.39 \\
\hline 7 & 0.47 & 0.18 & 0.29 & 0.59 & 0.27 & 0.50 & 2.07 & 1.36 \\
\hline 8 & 2.47 & 0.44 & 2.03 & 0.79 & 0.00 & 0.58 & 1.17 & 17.64 \\
\hline 9 & 0.19 & 0.07 & 0.12 & 0.57 & 0.46 & 0.34 & 0.26 & 0.22 \\
\hline 10 & 0.40 & 0.04 & 0.35 & 0.71 & 0.02 & 0.56 & 2.21 & 2.40 \\
\hline 11 & 0.78 & 0.13 & 0.66 & 0.82 & 0.18 & 0.54 & 1.44 & 4.42 \\
\hline 12 & 0.72 & 0.07 & 0.65 & 0.86 & 0.46 & 0.30 & 5.71 & 2.89 \\
\hline 13 & 0.32 & 0.01 & 0.26 & 0.72 & 0.46 & 0.52 & 1.73 & 0.40 \\
\hline
\end{tabular}


generates a Flu/(Flu + Pyr) ratio of $>0.40$ (Zhang et al. 2004). In the present study, the ratios of the $\Sigma C O M B / \Sigma E P A-$ PAHs ranged from 0.57 to 0.86 , which indicates that extensive combustion activities affected the PAHs in the sandstorm depositions. Furthermore the $\mathrm{An} /(\mathrm{An}+\mathrm{Phe})$ ratio ranged from 0 to 0.82 , with a median value of 0.25 and the Flu/(Flu + Pyr) ratio ranged from 0.30 to 0.58 with a median value of 0.50 . These findings further demonstrate that the PAHs originated from coal combustion sources.

The BaP/BghiP ratio can be used to distinguish traffic exhausts from coal combustion sources. For example, a $\mathrm{BaP} / \mathrm{BghiP}$ of $0.3-0.44$ indicates that the PAHs originated from auto exhaust, whereas a ratio of 0.9-6.6 indicates that the source was coal combustion (Sawicki 1962). Furthermore, a Flu/(Flu + Pyr) ratio of $<0.40$ is characteristic of crude liquid fossil fuel (traffic emission) combustion, whereas a ratio of $>0.4$ is characteristic of grass, wood or coal combustion (Zhang et al. 2004). In the present study, the ratio of $\mathrm{BaP} / \mathrm{BghiP}$ ranged from 0.26 to 5.58 , with a median value of 1.21 . In addition, $70 \%$ of the samples had a BaP/BghiP ratio of 0.9-6.6. Moreover, $70 \%$ of the Flu/ (Flu + Pyr) ratios were $>0.4$. As shown in Fig. 2, a plot of the $\mathrm{BaP} / \mathrm{BghiP}$ ratio versus the $\mathrm{Flu} /(\mathrm{Flu}+\mathrm{Pyr})$ ratio revealed that $62 \%$ of the samples fell within the combustion zone (I), which suggest that coal combustion is the primary source of the PAHs in the sandstorm depositions. However, 3 samples had a Flu/(Flu + Pyr) ratio of $<0.4$ and a $\mathrm{BaP} / \mathrm{BghiP}$ ratio of $<0.9$, which indicated that the source was traffic emission. Finally, 2 samples had a Flu/ (Flu + Pyr) ratio of $>0.4$ and a $\mathrm{BaP} / \mathrm{BghiP}$ ratio of $<0.9$, which indicates that the source was a mixture of coal combustion and traffic emission. Taken together, these results indicate that the primary combustion source was

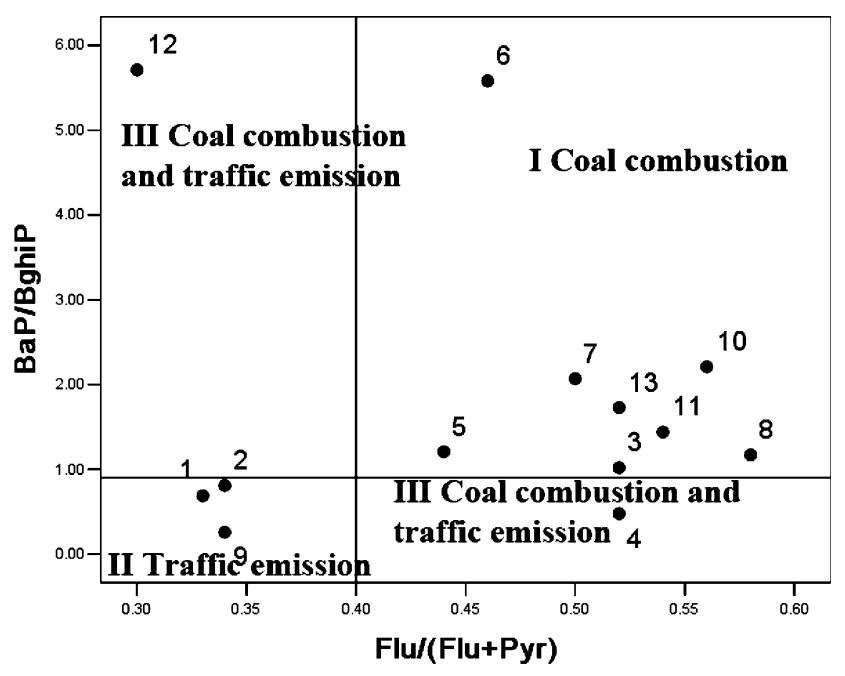

Fig. 2 PAH cross plot of the ratios of Flu/(Flu + Pyr) versus BaP/ BghiP in sandstorm deposition samples collected from urban areas of Beijing coal combustion, but that traffic emissions also influenced the presence of PAHs in the sandstorm depositions collected from urban areas in Beijing.

Another interesting finding of this study was that there was a general increase in PAHs concentrations from the northwest to the southeast in Beijing (Fig. 3). However, there was no significant correlation among the $\Sigma$ PAHs and minimum particle size $(r=0.10, p<0.05)$. Therefore, these findings may have occurred due to the location of the industrial zone, which is in southeast Beijing. If so, this would indicate that the major source of PAHs in Beijing was local energy consumption associated with industry. It has been shown that a large amount of waste gas or particles containing PAHs are emitted into the atmosphere as a result of incomplete combustion during industrial activities, and that these PAHs are subsequently transferred to soil via deposition (Trapido 1999). Taken together, these factors could explain the increased tendency of contamination from northwest to southeast in the urban areas of Beijing.

We used the nemerow composite index ( $p$ value), which is as follows, to assess the environmental quality of the samples collected in this study:

$P=\left\{\left[\left(P \mathrm{i}_{\mathrm{av}}\right)^{2}+\left(P \mathrm{i}_{\max }\right)^{2}\right] / 2\right\}^{1 / 2}$

where $P \mathrm{i}_{\mathrm{av}}$ is the average value of the individual pollutants indices and $P \mathrm{i}_{\max }$ is the maximum value of the individual pollutants indices (Ge et al. 2005). The values of 9 PAHs in the samples collected for this study were then compared to the target values specified for soils in the environmental standards of the Netherlands. The risk assessment indices used were Na, Phe, An, Flu, BaA, Chr, BaP, InP and BghiP

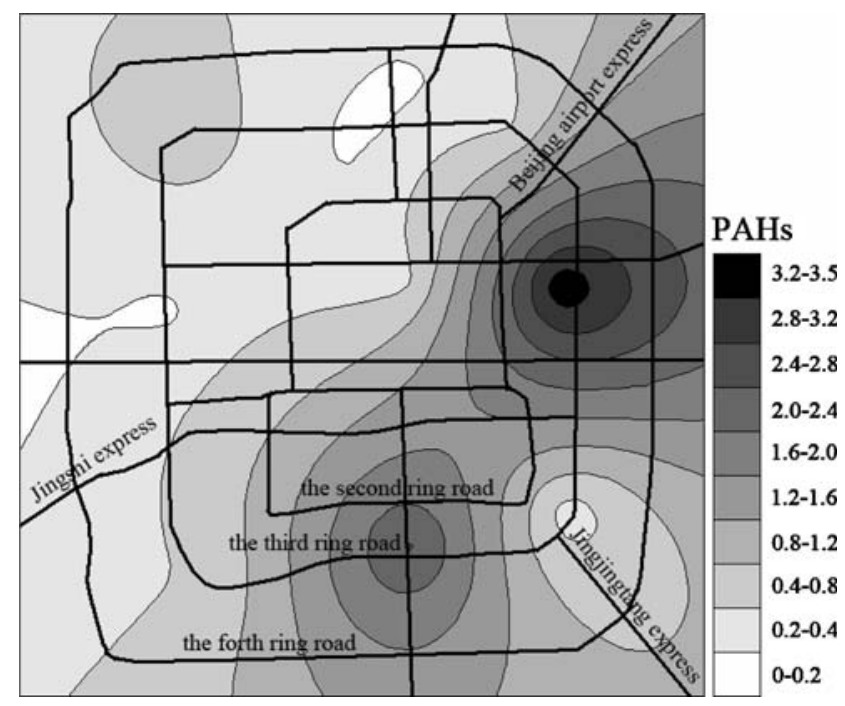

Fig. 3 Distribution of $\Sigma$ PAHs in urban areas of Beijing (data in the Figure are in $\mu \mathrm{g} \mathrm{g}^{-1}$ ) 
(Aannokkee 1990). The nemerow composite index ( $p$ value) may indicate the role of pollutants in the soil, especially the effect of high concentrations on soil environmental quality; therefore, it is commonly used to assess environmental pollution by PAHs (Ge et al. 2005). Because sandstorm depositions have a direct influence on surface soil, we used this standard to assess the PAHs in the sandstorm deposition. Based on the indices, environmental quality is divided into five environmental pollution grades, cleanness (safety) $(p \leq 0.7)$, warning value $(0.7<$ $p \leq 1.0)$, light pollution $(1.0<p \leq 2.0)$, middle high pollution $(2.0<p \leq 3.0)$ and heavy pollution $(p>3.0)$ (State Environmental Protection Administration of China (SEPA) 2004). The results revealed that the nemerow composite indices of the sandstorm deposition ranged from 0.22 to 17.64 (Table 1). Furthermore, $77 \%$ of the sandstorm deposition sampling sites had different grades of pollution and approximately $23 \%$ of these sites had a value of safety (cleanness). Because PAHs can act as endocrine disruptors, these findings indicate that it is necessary to monitor their status continuously.

Acknowledgment This study was supported by the National Natural Scientific Foundation of China (No. 20707031 and No. 20437020).

\section{References}

Aannokkee GJ (1990) MT-TNO research into the biodegradation of soils and sediments contaminated with oils and PAHs. In: World (ed) Contaminated soil, Kluwer Academic Publisher, New York

Bernal-Martinez A, Carrère H, Patureau D, Delgenes JP (2007) Ozone pre-treatment as improver of PAH removal during anaerobic digestion of urban sludge. Chemosphere 68:1013-1019

Blackburn GM, Kellard B (1986) Chemical carcinogens-Part II. Chem Ind 20:687-695
Budzinski H, Jones J, Bellocq J (1997) Evaluation of sediment contamination by polycyclic aromatic hydrocarbons in Gironde estuary. J Mar Chem 58:85-97. doi:10.1016/S0304-4203(97) 00028-5

Caia QY, Mo CH, Wu QT, Katsoyiannis A, Zeng QY (2008) The status of soil contamination by semivolatile organic chemicals (SVOCs) in China: a review. Sci Total Environ 389:209-224. doi:10.1016/j.scitotenv.2007.08.026

Durjava MK, ter Laak TL, Hermens JLM, Struijs J (2007) Distribution of PAHs and PCBs to dissolved organic matter: high distribution coefficients with consequences for environmental fate modeling. Chemosphere 67:990-997. doi:10.1016/j. chemosphere.2006.10.059

Franz TP, Eisenreich SJ, Swanson MB (1991) Evaluation of precipitation samplers for assessing atmospheric fluxes of trace organic contaminants. Chemosphere 23:343-361. doi:10.1016/ 0045-6535(91)90189-K

Ge CJ, An Q, Dong YH (2005) Residue and risk assessment of polycyclic aromatic hydrocarbons (PAHs) in soils around a steel mill. Rual Eco-Environ 21(2): 66-69, 73

Han T, Lin J, Wang Y, Zheng B, Song H, Liu J, Jiang G, Shi P, Zhang J (2007) Nature and control of "dust storms" in the BeijingTianjin region, China- a case study of the dust storm in Beijing on April 16, 2006. Bull Geol 26:117-127 (in Chinese)

Mielke HW, Wang W, Gonzales CR (2001) PAH and metal mixtures in New Orleans soils and sediments. Environ Sci Technol 281:217-227

Sawicki E (1962) Analysis of airborne particulate hydrons-their relative proportion affected by different types of pollution. $\mathbf{J}$ Polycyl Aromat Comp 22:23-25

Trapido M (1999) Polycyclic aromatic hydrocarbons in Estonian soil: contamination and frofiles. Environ Pollut 105:67-74. doi: 10.1016/S0269-7491(98)00207-3

Yang Y, Zhang XX, Korenaga T (2002) Distribution of polynuclear aromatic hydrocarbons fPAHs1 in the soil of Tokushima, Japan. Water Air Soil Pollut 38:51-60. doi:10.1023/A:1015517504636

Zhang ZL, Huang J, Yu G, Hong HS (2004) Occurrence of PAHs, PCBs and organochlorine pesticides in Tonghui River of Beijing, China. Environ Pollut 130:249-261. doi:10.1016/j.envpol.2003. 12.002 